\title{
DINÁMICA EMPRESARIAL EN VIZCAYA. 1914-1923. UNA APROXIMACIÓN
}

\author{
EDUARDO J. ALONSO OLEA
}

\section{INTRODUCCIÓN: MÉTODO Y FUENTES}

En este trabajo pretendemos esbozar la dinámica de las sociedades anónimas vizcaínas entre 1914 y 1923, centrándonos en sus resultados económicos y rentabilidad. Para ello contamos como fuente fundamental con los expedientes para el pago de la Tarifa $3^{a}$ de la Contribución de Utilidades de las propias empresas que se encuentran en el Archivo de la Diputación de Vizcaya ${ }^{1}$. No se sorprenda el lector porque en tal lugar se localicen materiales documentales que deberían estar en la Delegación de Hacienda. Como es sabido, la Diputación Provincial de Vizcaya, así como sus hermanas de Guipúzcoa y Álava, disfrutaban, gracias al Concierto Económico $^{2}$, de una amplia autonomía fiscal. Mediante un acuerdo destinado a establecer un periodo de adaptación desde el extinto régimen foral a la incorporación plena de las provincias vascongadas al régimen común —que como es bien

dministrativo de la Diputación Foral de Bizkaia. Hacienda. Utilidades Tarifa 3*. Liquidaciones de las Sociedades. Cajas 420-472. Como el impuesto de Utilidades no alcanzó efectivamente hasta el ejercicio de 1923 a las sociedades colectivas, comanditarias sin acciones y comunidades de bienes, hemos optado por simplificar este avance de resultados. Sin embargo sí habría que señalar que a a Echevas empresas relevantes dos notrieta y Larrínaga, que estaba) con un capital aportado de 50 millones en 1923. También cabría señalar Pocios (minas, astilleros, etc) cransformados metálicos de 3,5 millones de capital en 1923; Pedro P. Gandarias (minera) con 22 millones, o Chávarri Hnos., propietarios de minas con un millón de capital el el mismo año de 1923.

2 Alonso Olea (1995), passim. 
sabido no tuvo lugar, en el caso de Vizcaya y Guipúzcoa, hasta 1937-, datado en 1878 , y sus sucesivas renovaciones, las Diputaciones vascongadas se comprometían con el Ministerio de Hacienda a abonar un cupo en equivalencia de lo que el Ministerio recaudaría en las provincias. Para pagar tal cupo las Diputaciones vascongadas gestionaban un bloque de tributos - por eso llamados concertados- con entera libertad de su establecimiento, de sus mecanismos de cobro y de sus tarifas.

Pues bien, a resultas del acuerdo que indicamos, la Diputación vizcaína estableció, según sus necesidades y conveniencias, la Contribución sobre Utilidades en una fecha tan tardía como $1910^{3}$. Por lo tanto, el primer dato importante a indicar es que la fuente manejada es de índole fiscal, concretamente las liquidaciones provisionales y definitivas de la Tarifa $3^{a}$ de la Contribución de Utilidades, que grababa fundamentalmente los beneficios empresariales. Habida cuenta del tradicional fraude -ocultación según los contemporáneos- es difícil asegurar que los datos presentados sean absolutamente exactos; pero para nuestros fines son válidos en cuanto son todos de la misma procedencia y que, por lo tanto, la ocultación debería ser más o menos similar y alcanzaría, en el caso concreto de las sociedades anónimas, a los balances publicados en sus memorias. Hay que tener en cuenta que la inspección tributaria de la Diputación de Vizcaya, sobre todo desde los años finales de la década de los veinte, se mostró especialmente activa en comprobar que las liquidaciones del impuesto de Utilidades cuadraran con los balances de las empresas. Se localizan frecuentes liquidaciones definitivas desde 1921, pero en la mayoría de los casos no se puede hablar de modificación por fraude sino por errores de cálculo o interpretación de algunos apartados del Reglamento de la Diputación, con escasa incidencia real en cuanto a las cifras finales ${ }^{4}$.

'El establecimiento del tributo que nos ocupa fue paulatino, no sólo en las bases imponibles contempladas, que ya detallaremos, sino también con respecto a las sociedades afectadas. Comenzó siendo un tributo que alcanzaba en exclusiva a las Sociedades Anónimas, para, desde 1920, alcanzar también a las sociedades mineras, y desde 1923, gravar los beneficios de las sociedades regulares colectivas, comanditarias sin acciones y comunidades de bienes. Al año siguiente comenzó a gravar los notarios y a los corredores de bolsa. En 1934 alcanzó a las empresas individuales, futbolistas profesionales, academias y centros de enseñanzas, a las imprentas de libros. Sobre la contribución de utilidades vid. Reglamento (1901); Migoya (1910); Ministerio de la Gobernación (1913); Frigola Palao (1914); Ministerio de Hacienda (1920); Diputación de Álava (1927); Diputación de Álava (1928); Diputación Foral y provincia de Navarra (1928); Diputación de Guipúzcoa (1929); Ezcurdia (1933); Diputación de Vizcaya (1935).

${ }^{4}$ El contencioso más importante, por su volumen, fue el protagonizado por la Naviera Sota y Aznar, que recurrió todas las liquidaciones provisionales entre 1921 y 1926 porque la Diputación le sumaba al capital unas cantidades por unos fondos de seguros (de unos 60 millones de pesetas) que provocaba la duplicación del capital de la sociedad. En unos momentos en que los beneficios no alcanzaban tasas importantes respecto al capital social, la aplicación de la tarifa mínima (el $2 \%$ implicaba valores elevados. El recurso se ganó por la naviera y le tuvieron que devolver el dinero, por ello no aparece el total de las liquidaciones provisionales. Otro caso importante fue el de Aurrera, que entre 1924 y 
Un expediente de una empresa se estructuraba de la siguiente forma. A comienzos de cada año (la Diputación de Vizcaya tenía su ejercicio económico en consonancia con el año natural) se mandaban circulares a las sociedades anónimas para que enviaran su balance y memoria del año anterior. Con estos datos elaboraba una liquidación provisional y remitía a la empresa su resultado, con un plazo de 15 días para abonar el tributo correspondiente. Si había reclamación de la empresa o la inspección tributaria de la Diputación intervenía, se elaboraba una liquidación definitiva ${ }^{5}$.

La implantación del impuesto de Utilidades, fechada en 1908, data realmente del ejercicio de 1910, en que se comenzó a recaudar con escasos resultados. Entre 1911 y 1913 se fueron ampliando las comunicaciones a las sociedades, con un extraño paréntesis en 1913 en que desciende el número de las empresas que cuen$\tan$ con liquidación en ese año. Hay que tener en cuenta que la Diputación de Vizcaya, en ejercicio de sus facultades tributarias, estableció el impuesto con una tarifa menor que la del Ministerio de Hacienda y sobre otro concepto. Mientras que éste aplicaba el tributo sobre los beneficios totales de las sociedades - con tipos distintos según su actividad - aquélla lo hacía sobre los beneficios repartidos en forma de dividendos de las acciones, con un tipo fijo del $3 \%$; es decir, el dato sobre el que se gravaba el impuesto era el beneficio distribuido o los dividendos. Esto hace que las liquidaciones en muchos casos no existan puesto que, independientemente de los beneficios, hubo muchas empresas que no repartieron dividendo y quedaron de hecho exentas del impuesto. Además, no son extraños los casos en que con un simple certificado de la empresa sobre sus beneficios repartidos como dividendos la Diputación se conformara, por lo que no sabemos los datos ni de sus beneficios totales ni de su capital, puesto que el balance no consta ${ }^{6}$.

Desde 1914 se fijó la obligación de las sociedades de pagar el tributo sobre sus beneficios reales, con un porcentaje del $3 \%$, inferior al del Ministerio (que lo aplicaba con un tipo variable entre un $12,6 \%$ y un $0,5 \%$ según ramos de actividad). Al año siguiente se aumentó al 4\%, hasta 1920 en que se subió al 5\%. Desde 1921, a ejemplo de las modificaciones establecidas por el Ministerio de Hacienda en $1920^{7}$, se estableció un tipo variable en función del porcentaje que los beneficios repre-

1928 ocultó beneficios por cantidades entre 200.000 y 300.000 pesetas anuales, por lo que la Diputación le giró nuevas liquidaciones con recargos, aunque la apelación de la empresa sirvió para que se los devolvieran en 1936.

5 En los das que presentaremos seguidamente hemos tomado los datos de las liquidaciones provisionales y en el caso de que aparecieran posteriormente, de las definitivas, aunque insistimos en que la casos las diferencias fueron de poca monta a efectos recaudatorios.

- Sobre los beneficios que la política tributaria de la Diputación tenía para las empresas, y los contribuyentes en general, Alonso Olea (1994), pp. 261-86.

Ministerio de Hacieda (1920), passim. 
sentaban en el capital más reservas (tanto tácitas como explícitas) de las empresas, aunque también con un tipo menor al del Ministerio. Esta escala se modificó al alza en 1927.

Nosotros hemos obtenido los siguientes datos de las sociedades anónimas vizcaínas a efectos del pago del tributo de Utilidades. En primer lugar sus gastos de explotación y amortizaciones, sus ingresos totales, sus beneficios líquidos, el volumen de los mismos repartidos como dividendo (entre 1910 y 1913), su capital desembolsado y sus reservas desde 1921. La laguna entre 1910 y 1921 en cuanto al capital y reservas, que no consta en las liquidaciones, lo hemos tenido que solventar con la revisión de las cifras que figuran en los balances. Lógicamente, la aparición de nuevas empresas ampliaba el volumen de expedientes, al tiempo que su disolución hacía que dejaran de tributar y su expediente concluyera en un momento dado, por lo que es una fuente dinámica en el sentido de que desde 1910 podemos ver cuáles y cuántas se formaron, con la salvedad de algunos tipos como las mineras que no tributaron a la Diputación por Utilidades hasta 1921. Al mismo tiempo, agrupadas por sectores, podremos ver resultados parciales en determinadas actividades económicas, o llegado el caso en qué momentos se formaron más sociedades de un determinado sector o a la inversa en qué momentos desaparecieron y ver el efecto de la coyuntura en distintos sectores.

Para terminar este apartado hay que advertir que la consulta de la fuente no es completa todavía. Los expedientes empresariales se interrumpian en cuanto se alcanzaba un volumen de papel determinado y pasaban los años siguientes a otro expediente que en muchos casos todavia no hemos tenido oportunidad de revisar. Hoy por hoy tenemos datos ultimados y elaborados del período 1910-1923, sin que ello quiera decir que no dispongamos de información de ejercicios siguientes. Al estructurarse la fuente según comienzo de actividad de las sociedades, nos faltarían datos de las sociedades formadas desde 1923 y piezas restantes de las empresas formadas antes de tal año. En resumen, tenemos todos los datos empresariales entre 1914 y 1923 y parcialmente desde 1924 hasta 1936. La laguna más importante, en el período en que las segundas piezas son más abundantes, se situaría en los años 30 , tanto por efecto del volumen de información manejado, como por la actividad del Jurado Mixto de Utilidades ${ }^{8}$, así como por la abundancia de liquidaciones definitivas derivadas de una mucho mayor diligencia de la inspección tributaria provincial.

${ }^{8}$ El Jurado Mixto de Utilidades se creó en 1927, a resultas de la renovación del Concierto de 1926, y se encargaba de adjudicar a cada territorio la parte de negocio de cada empresa y así recaudar, cada administración de Hacienda, su tributo correspondiente. Se formaron tres Jurados provinciales, y uno Central ocupado de solventar las discrepancias de los provinciales. La actuación de unos y otros ralentizó mucho las liquidaciones de las sociedades, por lo que desde 1927 hay muchos huecos por comple tar, que cuando acabemos la serie se colmatarán. 


\section{EVOLUCIÓN DE LOS RESULTADOS EMPRESARIALES DE LAS SOCIEDADES ANÓNIMAS VIZCAÍNAS. 1914-1923.}

Antes de comenzar con el análisis de los datos arriba propuestos hay que puntualizar varios extremos. En primer lugar diremos que, debido a la singular aplicación del Concierto Económico, las sociedades creadas antes de 1900 tributaban por todos sus beneficios a la Diputación de Vizcaya. Las formadas posteriormente - salvo las navieras-, en principio, debían hacerlo a la Diputación por sus beneficios obtenidos dentro de la provincia y al Ministerio de Hacienda respecto a los de territorio común. Pues bien, vemos cómo tanto unas como otras abonaron sus tributos fundamentalmente a la Diputación vizcaína hasta 1927, momento en el cual comenzó a funcionar el Jurado mixto de Utilidades que hacía un reparto de los beneficios - y por lo tanto de la parte que tributaba por los mismos- entre territorio concertado y común. Ello no evita que en algunos casos antes de 1927 se hicieran algunos cálculos aproximados de los beneficios obtenidos fuera y sólo de ellos pagara a la Diputación, y a la inversa veremos sociedades que aunque con domicilio en territorio común pagaban a la Diputación de Vizcaya por los beneficios obtenidos por su sucursal ahí radicada. En estos casos, en que los beneficios totales no eran los utilizados para el cálculo del impuesto sino sólo los obtenidos en la provincia, hemos empleado estos últimos, sin obviar el dato concreto de los beneficios totales que sí nos pueden interesar por otras razones. Siendo nuestro trabajo sobre los beneficios empresariales de las sociedades vizcaínas ${ }^{9}$, nos centraremos fundamentalmente en esta variable, para mantener la homogeneidad de la serie.

Otra referencia habría que hacer acerca de las sociedades extranjeras. Como en muchos casos las reuniones del consejo y de la junta general se hacían en el extranjero sus memorias y sus balances se editaban con valores establecidos en sus respectivas monedas (libras esterlinas, francos franceses, coronas noruegas o francos belgas); por eso, también hay que contemplar el cambio con la peseta pues podría darse el caso, como se dio en alguna, de que en moneda extranjera el resultado fuera creciente pero en pesetas - por el cambio- resultara contrario (por ejemplo, Tramways et Electricitè de Bilbao, explotadora del tranvía urbano de Bilbao). En todos estos casos la propia Diputación evaluaba, sobre el balance y la memoria, los beneficios de la sucursal radicada en Vizcaya y sobre ellos pagaba la sociedad, resultando éstos los elegidos para nuestros cómputos. En estos dos casos, empresas

\footnotetext{
${ }^{9}$ Hay algún caso en que el término empresa vizcaina es harto discutible puesto que aparecen también sociedades que no tenían en Vizcaya más que su domicilio social y una pequeña oficina -a veces ni eso- con el fin de evitar el pago de impuestos.
} 
con actividades fuera y las extranjeras, la Diputación calculaba, en proporción de los beneficios obtenidos, la parte del capital que correspondería a Vizcaya, y sobre ambos guarismos se hacía el cálculo de tributo. Sobre estas estimaciones de capital, pues, hemos establecido también nuestros cálculos de capital y reservas.

En relación directa con los datos localizados presentamos en estos momentos referencias de un total de 270 sociedades anónimas y comanditarias por acciones, la mayoría de ellas con domicilio social en Vizcaya, más algunas con domicilio fuera, tanto en el resto del país como en el extranjero. A tenor de los datos que presentamos en el Cuadro 1 caben hacer diversas consideraciones. En primer lugar sobre el proceso de formación de sociedades anónimas. Entre 1914, primer año del que tenemos datos fidedignos, y 1921 se doblarían en número, para volver a incrementarse en 1923. El hecho de que entre 1920 y 1921 aumenten en número casi en cincuenta empresas se debe, sobre todo, a la incorporación de las empresas mineras a nuestros datos. Hay que tener en cuenta que entre uno y otro año se suman trece empresas mineras a las que ya tributaban a la Diputación vizcaína. Aun así el incremento de empresas es real, por lo que el proceso de formación de sociedades queda bien patente.

Si fijamos nuestra atención a los datos de la segunda y tercera columna, en que apuntamos los totales de beneficios y de capital más reservas, se puede ver fácilmente cómo el punto álgido de los primeros fue el último de la guerra mundial, 1918, para descender de forma acusada los dos años siguientes, resultando los beneficios de los tres últimos años de la serie bastante más modestos. En cambio, respecto al capital, si bien durante 1918 y 1919 aumenta de forma importante alcanza su máximo en 1920, para luego descender entre 1921 a 1923. La explicación es sencilla de apuntar. Por una parte contamos con un número mayor de sociedades, por lo tanto es lógico que los capitales aumentaran, pero además también se explica por el incremento de las reservas debido a los grandes beneficios obtenidos durante la guerra. Con la crisis de posguerra las reservas servirán para absorber las pérdidas y por eso descienden en valores absolutos.

Semejantes consideraciones se pueden hacer si contemplamos los datos de las columnas 5 y 6 del Cuadro 1 . Si dividimos los beneficios totales entre el número de empresas también observamos cómo el valor más importante se alcanza en 1918, para descender de forma acusada desde aquí hasta 1921 en que se mantienen en niveles levemente superiores a los de 1914. Respecto al capital más reservas volvemos a encontrar su máximo por empresa en 1920 , pero con una acusada caída en 1921, debido, como decíamos más arriba, al incremento de sociedades estudiadas y a la pérdida de reservas.

Es ampliamente conocido el proceso de incremento de precios sufrido por España en los años de la primera guerra mundial. Por ello hemos deflactado los 


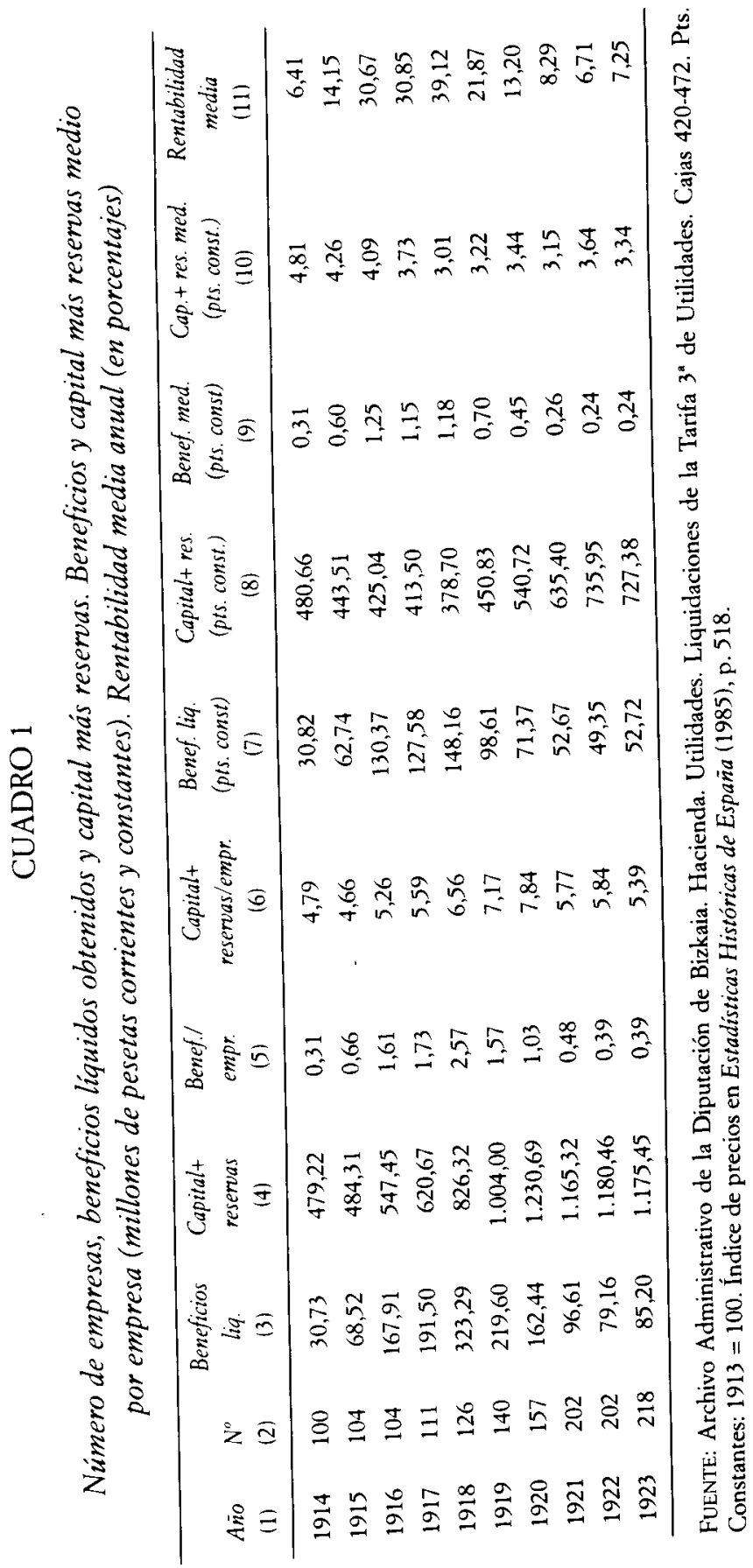


datos que hasta ahora presentamos en términos corrientes para hacerlos en constantes y ver si realmente estos fuertes incrementos de beneficios y capitales se aparejaron con la subida de precios. Pues bien, si observamos los datos de las columnas 7 y 8 cabe corroborar lo dicho más arriba. En cuanto a los beneficios líquidos su incremento fue bastante más moderado. Entre 1916 y 1919 es innegable un incremento de beneficios, aunque en términos constantes los obtenidos entre 1921 y 1923 se situaron por debajo de los de 1915. Respecto al capital y reservas cabe decir otro tanto, puesto que el máximo se situaría no en 1920 sino en 1922 , con el mínimo precisamente en el año en que se obtuvieron mayores beneficios (1918). El fuerte incremento de precios durante la guerra aminoró de forma importante los beneficios de las sociedades anónimas vizcaínas en términos reales. $\mathrm{Si}$ observamos ahora los datos de las columna 9 y 10 donde presentamos los beneficios y capitales medios por empresa en valores constantes. Respecto a los beneficios volvemos a encontrar el aumento esperado en los años de la guerra mundial, aunque ya no destaca 1918 sino que se muestran bastante uniformes entre 1916 a 1918, para desde aquí descender de forma acusada hasta 1921 a 1923 en que no se alcanzan los beneficios medios de 1914. El mismo efecto de reducir los valores se puede indicar respecto al capital y reservas puesto que el máximo se sitúa en 1914 en un proceso descendente hasta 1918, punto a partir del cual se mantienen más o menos uniformes.

Por último comentaremos los valores calculados de rentabilidad (relación de los beneficios sobre el capital más reservas en términos porcentuales). En este caso es claro que el máximo de rentabilidad se alcanza en 1918, casi el $40 \%$, luego desciende de forma acusada hasta 1921, marcando un mínimo en 1922, sólo levemente superior a 1914, para volver a crecer en 1923.

En resumen, creemos que todos estos datos muestran dos hechos. Por una parte, aunque se constata el aumento de beneficios correspondientes a los años de la guerra mundial, sobre todo al ejercicio de 1918 , también es cierto que no fueron tan elevados como su simple montante absoluto muestra. Por otra, manifiestan la gravedad de la crisis de posguerra de la economía vizcaína. Naturalmente, al ser estos datos agregados de todas las empresas existen variantes sectoriales que seguidamente comentaremos.

\section{BENEFICIOS Y RENTABILIDAD POR SECTORES INDUSTRIALES}

En el Cuadro 2 desglosamos la rentabilidad financiera (beneficios líquidos sobre el capital más reservas) entre 1914 y 1923 , que son los datos definitivos de las sociedades anónimas vizcaínas. De estos datos llaman la atención ciertos valores. En 


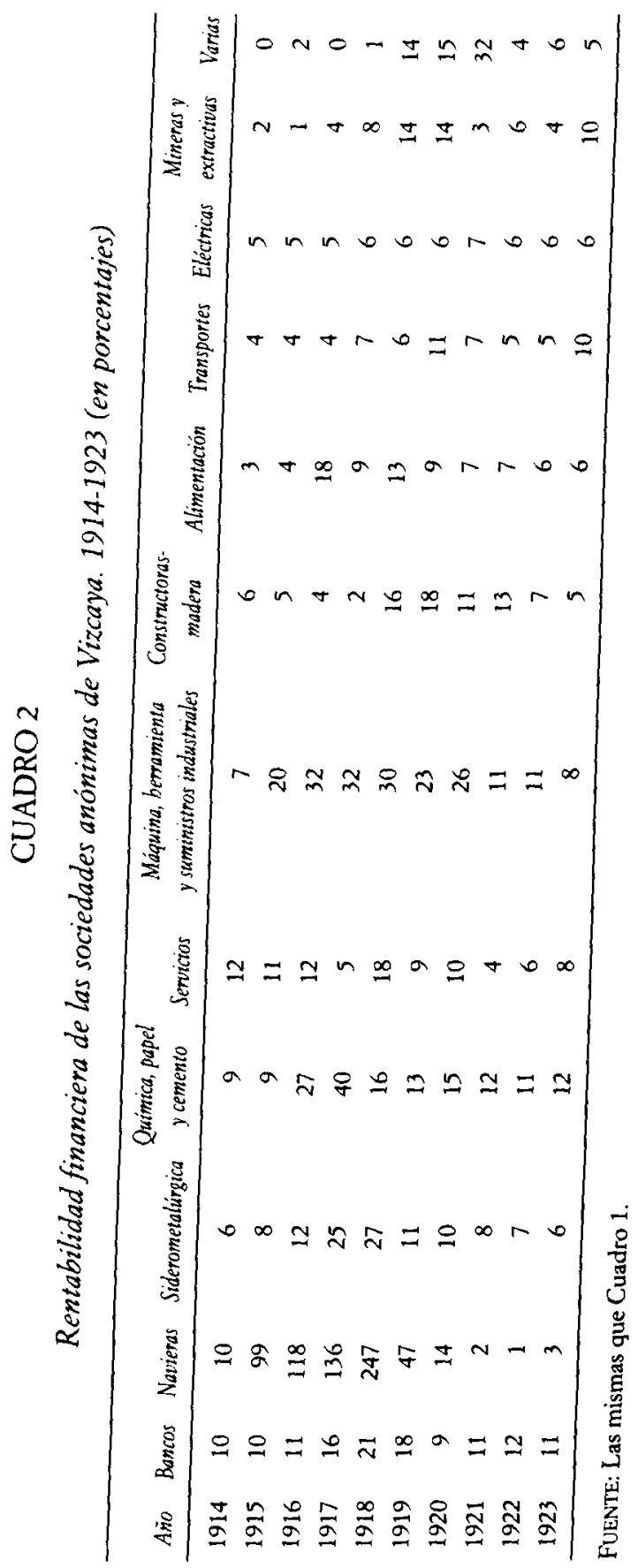


unos casos debido a condiciones reales y en otros a la falta de información, como sucede con los valores de las sociedad mineras y extractivas, que hasta 1921 pagaron el impuesto al Ministerio de Hacienda, con lo que sólo tenemos para las grandes sociedades de la minería del hierro vizcaína.

Seguidamente haremos unos breves comentarios sobre la rentabilidad media de cada sector y su relación con la media general anual (última columna del Cuadro 1). En relación con los Bancos (Apéndice, Cuadro 1), arrojan un máximo de beneficios en términos absolutos en 1919, aunque porcentualmente lo hacen en 1918, doblando sus beneficios entre 1916 y 1917, lo que nos parece un reflejo de la coyuntura favorable de la guerra mundial, para comenzar a descender desde 1919 y alcanzar el mínimo al año siguiente, aunque se recuperaron en los siguientes. Fue desde luego uno de los sectores más rentables y más beneficiados por la coyuntura alcista. Al mismo tiempo, visto respecto a la rentabilidad media anual, sus resultados no fueron tan exagerados como en otros sectores, aunque en los años de normalidad se mantuvieron por encima de la media, pese a descender su rentabilidad a la mitad entre 1919 y 1920.

El sector más aventajado en cuanto a beneficios y rentabilidad será el negocio naviero (Apéndice, Cuadro 2). Las empresas navieras no sólo se beneficiaron de la coyuntura alcista de los fletes, o de la condición de nación neutral, sino también obtuvieron beneficios suplementarios por la venta a precios exorbitados de buques, además del beneficio que supuso para los balances de distintas empresas las indemnizaciones de los seguros por la pérdida de barcos fuera torpedeados o por naufragios. Cuando la guerra acabó se vieron afectadas por una general recesión en el mercado marítimo, por lo que sus beneficios cayeron en picado. Las que habían previsto la situación o incrementado sus reservas pudieron hacer frente a la crisis; las que no lo habian hecho desaparecieron. Pero hubo empresas que no incrementaron tanto sus beneficios o que destinaron a reservas buena parte de ellos, de modo que, tras los años malos de la posguerra pudieron mantener un volumen de ganancias, si no tan exagerado sí apreciable.

En términos más modestos, pero también importantes debido a su peso específico en la economía provincial y por sus capitales, se sitúan las siderometalúrgicas (Apéndice, Cuadro 3), que con volúmenes menos exagerados presencian también un alza importante de su rentabilidad en los años de la guerra para volver a índices de preguerra tras ella, aunque en este caso también influyeron los conflictos huelguísticos que protagonizaron sus trabajadores en esos años. Estas sociedades, sólo en 1914 y 1922 se situarían por encima de la media en cuanto a su rentabilidad. Sus capitales, al contrario de los erráticos beneficios, experimentaron un continuo incremento; desde 1921 tienden a mantenerse en torno a los 180 millones de pesetas. 
En el grupo formado por las sociedades químicas, papeleras y cementeras (Apéndice, Cuadro 4) observamos una tendencia en cuanto a su rentabilidad similar: subida en la guerra mundial, con caída en los primeros años veinte. Presentan, por contraste, unos totales de beneficios y capitales más reservas máximos en 1920 , aunque descienden desde este año de forma significativa.

Dentro de los servicios (Apéndice, Cuadro 5), empresas de variada dedicación (espectáculos, editoriales, etc.), destaca la modestia de sus beneficios en términos absolutos. En el punto álgido de beneficios de los demás sectores, van a tener un modesto $5 \%$ de rentabilidad media, en posiciones cercanas a la media, con la salvedad de 1914 en que casi la doblan, pero adelantan su «crisis» a 1921. Sus capitales experimentan un continuo aumento durante todo el período estudiado, con un repunte en 1920, por la incorporación a los datos de una empresa francesa de importante capital (Establecimientos Debray), aunque no tendrá continuidad.

En el caso del variado grupo que comentamos seguidamente, el que genéricamente se ocuparía de la fabricación de máquina herramienta, astilleros y suministros industriales (Apéndice, Cuadro 6), encontramos también la tendencia a una mayor rentabilidad con la coyuntura alcista de la guerra mundial, y una caída de la misma desde 1921, por consideraciones semejantes a las que hicimos cuando hablamos de las siderometalúrgicas, con las que están evidente y estrechamente relacionadas. En cuanto a sus beneficios, tienen dos máximos en 1918 y 1920 con un descenso desde aquí hasta 1923. Más importante es el descenso de sus fondos propios que desde el máximo de 1920 disminuyen en unos 14 millones, para recuperarse en los dos últimos ejercicios.

En referencia a las constructoras (Apéndice, Cuadro 7), se puede decir que no arrojan una rentabilidad como los sectores hasta aquí vistos, sino que se mantienen en un nivel modesto. Sus beneficios totales sí van a reflejar, a escala, la coyuntura alcista de la guerra mundial, pero con retraso, resultando sus mejores ejercicios los siguientes a 1917 , aunque hay que tener presente que también aumentan las empresas de la muestra, hasta alcanzar el máximo en 1922. Semejantes consideraciones se pueden hacer respecto a los cómputos de capital y reservas.

Para las sociedades dedicadas al ramo de la alimentación (Apéndice, Cuadro 8) vemos en general una tendencia al crecimiento de su rentabilidad durante los años de la guerra, por supuesto por debajo de la media, para mantenerse en un nivel que rondaría la media de todos los sectores en los años finales de la serie. Pese a los importantes capitales invertidos, con un máximo en 1919, sus beneficios totales no se puede decir que sean importantes. Aunque hay que tener en cuenta en este sector habría sólo un puñado de empresas de importancia (Harino Panadera, Bodegas 
Bilbaínas o CVNE), mientras que otras no dejarían de ser modestas fábricas de dulces o lácteos. También, por ello, los beneficios se concentrarían más en las empresas más relevantes teniendo resultados, aunque proporcionalmente no inferiores, más modestos.

El sector de los transportes (Apéndice, Cuadro 9), con una mayoría de empresas ferroviarias, contaba con unos capitales considerables. Se situó durante todo el período, menos en 1923, por debajo de la media en cuanto a su rentabilidad. Llama atención cómo no se beneficiaron de la coyuntura alcista de la guerra mundial, pues se mantuvieron en el $4 \%$ entre 1914 y 1916, para subir levemente hasta el 7\% al año siguiente y volver al 6\% en 1918. Será en 1919 cuando casi doblen su rentabilidad, para volver a un nivel modesto en 1923. La importancia de los capitales empleados (80-90 millones) no se correspondió en modo alguno con su rentabilidad, siendo la excepción la que tenía beneficios de significación.

En relación a las sociedades eléctricas (Apéndice, Cuadro 10), se pueden hacer consideraciones semejantes, pues se mantendrían en una rentabilidad entre el 5 y el $6 \%$, salvo 1920 en que llegan hasta una media del $7 \%$, muy lejos, sobre todo en los años de la guerra mundial, de los valores medios anuales. Sí muestra, por contraste con otras ramas, un incremento continuo de beneficios y capital de forma que será en 1923 cuando alcancen sus valores máximos.

Las consideraciones que podamos hacer sobre el sector minero (Apéndice, Cuadro 11) son parciales por el motivo avanzado más arriba. Abonaron el Impuesto de Utilidades en su Tarifa $3^{a}$ a la Diputación desde 1921 en razón del encabezamiento del Impuesto de Mineral, que se descontaba para el pago de las Utilidades por lo que junto al uno debió de encabezarse —en este sector- la otra. Sin embargo tenemos una serie de sociedades mineras y de ejercicios suficiente para que, aunque sea aproximadamente, podamos hacer algunas consideraciones. Respecto a las sociedades anónimas ${ }^{10}$ mineras dedicadas a la extracción de hierro, de larga tradición en Vizcaya, no encontramos beneficios espectaculares. Habían llegado ya los años de rendimientos decrecientes, problemas de mercado y competencia. Sufrieron, como efecto de la crisis de posguerra, problemas de demanda de la siderurgia británica y nacional, por lo que sus rendimientos no se recuperaron hasta $1923^{11}$.

${ }^{10}$ Remarcamos el término Sociedades Anónimas porque habia otras importantes empresas dedicadas al negocio minero (propietarias, arrendatarias o comercializadoras) que estaban organizadas como colectivas o comunidades de bienes, como Echevarrieta y Larrínaga, Chávarri Hermanos o Pedro P. de Gandarias.

1 Para ver, por contraste, la rentabilidad que arrojaban estas sociedades hasta el cambio de siglo, vid. Montero, Manuel (1995). 
Dentro del apartado de «varios» (Apéndice, Cuadro 12) contamos con sociedades de difícil o imposible adscripción a los apartados anteriores. Se puede resumir su situación diciendo que entre 1914 y 1917 tuvieron una rentabilidad mínima; cuando la tuvieron, entre 1918 a 1920, aumentaron bastante su rentabilidad, aunque sólo se situarían por encima de la media en 1920, para volver, en los tres últimos años de la serie a beneficios más modestos, por debajo de la media, pero superando sus resultados anteriores a 1919. Sí muestran, por contraste, un incremento continuo de capital con máximos en 1921 y 1922, aunque su máximo en beneficios totales lo lograron en 1920.

\section{CONCLUSIONES}

Hemos hecho un repaso somero por necesidad de espacio y por provisionalidad de los datos. Por una parte, el fuerte impacto en los beneficios empresariales de la guerra mundial de 1914-1918, y el reflejo de la crisis subsiguiente en los primeros años de la década de los veinte. Sin embargo, no nos deben de llamar a engaño las cifras. Hubo sectores que no se beneficiaron en nada, o casi nada, de la coyuntura alcista (eléctricas, transportes o alimentación) siendo sobre todo las navieras, las siderometalúrgicas, las empresas de bienes de equipo o los bancos, los grandes beneficiados. A la inversa, cuanto mayores fueron los beneficios obtenidos entre 1914 a 1918, también mayores fueron los problemas de posguerra, no superados en general hasta 1923.

También creemos que nuestras cifras patentizan la interrelación económica entre distintos sectores y su relación mayor o menor, con la coyuntura económica mundial. De cualquier forma nos parece suficientemente interesante y válido el trabajo que presentamos puesto que individualiza - con las limitaciones apuntadas- las distintas coyunturas sectoriales, lo que nos permite conocer mejor la dinámica empresarial vizcaína en el período contemplado. Por último, diremos que en la actualidad ya contamos con datos completos para el período 1910-1935, con un total de 608 empresas, por lo que en un plazo no muy lejano esperamos poder presentar una serie más amplia que permita establecer de forma más completa la dinámica empresarial de Vizcaya durante estos 25 años. Así, veremos cómo se produjo la recuperación económica de los años veinte y el terrible efecto que tuvo la crisis de los años treinta en la economía vizcaina. 


\section{APÉNDICE}

\section{CUADRO 1}

Bancos 1914-1923

\begin{tabular}{ccccc}
\hline Año & $N^{\circ}$ & Beneficios liquidos & Capital + reservas & Ben $/ c .+r$ \\
\hline 1914 & 4 & 5,62 & 56,69 & $10 \%$ \\
1915 & 4 & 5,63 & 55,39 & $10 \%$ \\
1916 & 4 & 6,71 & 57,19 & $11 \%$ \\
1917 & 5 & 14,27 & 63,65 & $16 \%$ \\
1918 & 6 & 34,57 & 108,41 & $21 \%$ \\
1919 & 8 & 47,35 & 210,72 & $18 \%$ \\
1920 & 8 & 33,62 & 322,01 & $9 \%$ \\
1921 & 11 & 32,47 & 327,41 & $11 \%$ \\
1922 & 11 & 33,57 & 330,17 & $12 \%$ \\
1923 & 11 & 34,13 & 332,56 & $11 \%$ \\
\hline
\end{tabular}

\section{CUADRO 2}

Navieras 1914-1923

\begin{tabular}{ccccc}
\hline Año & $N^{\circ}$ & Beneficios líquidos & Capital + reservas & Ben/c.+r. \\
\hline 1914 & 22 & 4,69 & 57,50 & $10 \%$ \\
1915 & 23 & 38,00 & 56,61 & $99 \%$ \\
1916 & 19 & 124,65 & 101,24 & $118 \%$ \\
1917 & 19 & 123,82 & 130,32 & $136 \%$ \\
1918 & 19 & 220,56 & 210,27 & $247 \%$ \\
1919 & 23 & 117,87 & 239,46 & $47 \%$ \\
1920 & 21 & 49,60 & 224,75 & $14 \%$ \\
1921 & 21 & 7,58 & 165,27 & $2 \%$ \\
1922 & 18 & 2,44 & 150,98 & $1 \%$ \\
1923 & 19 & 1,83 & 140,42 & $3 \%$ \\
\hline
\end{tabular}

\section{CUADRO 3}

Siderometalurgicas 1914-1923

\begin{tabular}{crccc}
\hline Año & $N^{\circ}$ & Beneficios liquidos & Capital + reservas & Ben $/ c+r$. \\
\hline 1914 & 9 & 9,46 & 104,72 & $6 \%$ \\
1915 & 9 & 12,40 & 107,28 & $8 \%$ \\
1916 & 9 & 16,94 & 114,72 & $12 \%$ \\
1917 & 9 & 23,30 & 116,84 & $25 \%$ \\
1918 & 10 & 25,75 & 162,77 & $27 \%$ \\
1919 & 10 & 16,90 & 173,34 & $11 \%$ \\
1920 & 11 & 29,18 & 193,31 & $10 \%$ \\
1921 & 12 & 16,23 & 182,04 & $8 \%$ \\
1922 & 13 & 10,38 & 186,38 & $7 \%$ \\
1923 & 13 & 15,28 & 187,20 & $6 \%$ \\
\hline
\end{tabular}




\section{CUADRO 4}

Quimicas, papeleras y cementeras 1914-1923

\begin{tabular}{rrrrr}
\hline Año & $N^{\circ}$ & Beneficios liquidos & Capital + reservas & Ben/c.+r \\
\hline 1914 & 9 & 3,52 & 90,54 & $9 \%$ \\
1915 & 8 & 3,75 & 92,22 & $9 \%$ \\
1916 & 9 & 8,19 & 93,87 & $27 \%$ \\
1917 & 11 & 16,81 & 127,97 & $40 \%$ \\
1918 & 14 & 21,59 & 149,09 & $16 \%$ \\
1919 & 15 & 17,58 & 172,67 & $13 \%$ \\
1920 & 18 & 27,00 & 213,47 & $15 \%$ \\
1921 & 25 & 22,63 & 158,19 & $12 \%$ \\
1922 & 22 & 16,64 & 161,34 & $11 \%$ \\
1923 & 28 & 16,28 & 152,90 & $12 \%$ \\
\hline
\end{tabular}

\section{CUADRO 5}

Servicios 1914-1923

\begin{tabular}{ccccc}
\hline Año & $N^{\circ}$ & Beneficios liquidos & Capital + reservas & Ben $/ c+r$. \\
\hline 1914 & 6 & 0,14 & 1,84 & $12 \%$ \\
1915 & 7 & 0,15 & 2,87 & $11 \%$ \\
1916 & 7 & 0,18 & 2,97 & $12 \%$ \\
1917 & 9 & 0,17 & 4,02 & $5 \%$ \\
1918 & 12 & 0,50 & 3,86 & $18 \%$ \\
1919 & 14 & 0,40 & 6,10 & $9 \%$ \\
1920 & 18 & 0,90 & 27,40 & $10 \%$ \\
1921 & 26 & 0,35 & 6,98 & $4 \%$ \\
1922 & 28 & 0,57 & 8,69 & $6 \%$ \\
1923 & 29 & 0,91 & 15,22 & $8 \%$ \\
\hline
\end{tabular}

\section{CUADRO 6}

Máquina berramienta, astilleros y suministros industriales 1914-1923

\begin{tabular}{ccccc}
\hline Año & $N^{\prime \prime}$ & Beneficios liquidos & Capital + reservas & Ben $/ c+r$ \\
\hline 1914 & 9 & 0,89 & 39,97 & $7 \%$ \\
1915 & 11 & 1,71 & 40,25 & $20 \%$ \\
1916 & 11 & 2,82 & 40,49 & $32 \%$ \\
1917 & 11 & 4,29 & 41,81 & $32 \%$ \\
1918 & 13 & 8,01 & 46,31 & $30 \%$ \\
1919 & 15 & 4,51 & 49,55 & $23 \%$ \\
1920 & 18 & 7,49 & 54,34 & $26 \%$ \\
1921 & 25 & 3,07 & 31,00 & $11 \%$ \\
1922 & 25 & 2,40 & 34,28 & $11 \%$ \\
1923 & 28 & 1,61 & 39,79 & $8 \%$ \\
\hline
\end{tabular}


CUADRO 7

Constructoras 1914-1923

\begin{tabular}{ccccc}
\hline Año & $N^{\circ}$ & Beneficios liquidos & Capital + reservas & Ben $/ c+r$ \\
\hline 1914 & 2 & 0,19 & 3,98 & $6 \%$ \\
1915 & 2 & 0,10 & 3,98 & $5 \%$ \\
1916 & 2 & 0,08 & 4,00 & $4 \%$ \\
1917 & 1 & 0,07 & 3,06 & $2 \%$ \\
1918 & 3 & 0,87 & 4,27 & $16 \%$ \\
1919 & 5 & 0,86 & 5,59 & $18 \%$ \\
1920 & 5 & 0,78 & 7,79 & $11 \%$ \\
1921 & 7 & 1,13 & 7,81 & $13 \%$ \\
1922 & 7 & 0,61 & 10,50 & $7 \%$ \\
1923 & 6 & 0,51 & 9,08 & $5 \%$ \\
\hline
\end{tabular}

\section{CUADRO 8}

Aimentación 1914-1923

\begin{tabular}{rrccr}
\hline Año & $N^{\circ}$ & Beneficios liquidos & Capital + reservas & Ben $/ c+r$. \\
\hline 1914 & 8 & 0,78 & 12,87 & $3 \%$ \\
1915 & 8 & 0,98 & 13,16 & $4 \%$ \\
1916 & 8 & 1,30 & 11,98 & $18 \%$ \\
1917 & 8 & 1,15 & 13,73 & $9 \%$ \\
1918 & 9 & 2,14 & 14,14 & $13 \%$ \\
1919 & 10 & 2,26 & 16,91 & $9 \%$ \\
1920 & 11 & 1,95 & 32,37 & $7 \%$ \\
1921 & 15 & 2,16 & 24,26 & $7 \%$ \\
1922 & 17 & 1,72 & 30,36 & $6 \%$ \\
1923 & 17 & 1,77 & 26,83 & $6 \%$ \\
\hline
\end{tabular}

\section{CUADRO 9}

Transportes 1914-1923

\begin{tabular}{ccccc}
\hline Año & $N^{o}$ & Beneficios liquidos & Capital + reservas & Ben $/ c+r$ \\
\hline 1914 & 13 & 3,71 & 83,94 & $4 \%$ \\
1915 & 14 & 3,99 & 85,40 & $4 \%$ \\
1916 & 14 & 4,54 & 85,15 & $4 \%$ \\
1917 & 14 & 4,53 & 81,74 & $7 \%$ \\
1918 & 14 & 4,36 & 82,79 & $6 \%$ \\
1919 & 14 & 6,58 & 85,02 & $11 \%$ \\
1920 & 15 & 6,86 & 90,18 & $7 \%$ \\
1921 & 15 & 5,63 & 88,71 & $5 \%$ \\
1922 & 15 & 5,93 & 90,25 & $5 \%$ \\
1923 & 16 & 6,22 & 91,04 & $10 \%$ \\
\hline
\end{tabular}




\section{CUADRO 10}

Eléctricas 1914-1923

\begin{tabular}{ccccc}
\hline Año & $N^{\circ}$ & Beneficios liquidos & Capital + reservas & Ben $/ c+r$. \\
\hline 1914 & 14 & 1,70 & 24,46 & $5 \%$ \\
1915 & 14 & 1,71 & 24,76 & $5 \%$ \\
1916 & 15 & 1,99 & 28,34 & $5 \%$ \\
1917 & 15 & 2,15 & 29,44 & $6 \%$ \\
1918 & 15 & 2,56 & 29,75 & $6 \%$ \\
1919 & 15 & 2,75 & 29,92 & $6 \%$ \\
1920 & 15 & 3,17 & 30,52 & $7 \%$ \\
1921 & 15 & 2,96 & 30,17 & $6 \%$ \\
1922 & 16 & 2,98 & 32,67 & $6 \%$ \\
1923 & 16 & 3,63 & 35,15 & $6 \%$ \\
\hline
\end{tabular}

\section{CUADRO 11}

Mineras y extractivas 1914-1923

\begin{tabular}{ccccc}
\hline Año & $N^{\circ}$ & Beneficios liquidos & Capital + reservas & Ben/c. $+r$ \\
\hline 1914 & 1 & 0,01 & 0,38 & $2 \%$ \\
1915 & 1 & 0,00 & 0,38 & $1 \%$ \\
1916 & 2 & 0,37 & 4,74 & $4 \%$ \\
1917 & 2 & 0,64 & 4,38 & $8 \%$ \\
1918 & 2 & 1,99 & 8,38 & $14 \%$ \\
1919 & 2 & 1,99 & 8,38 & $14 \%$ \\
1920 & 3 & 0,30 & 16,99 & $3 \%$ \\
1921 & 16 & 2,33 & 129,32 & $6 \%$ \\
1922 & 15 & 1,39 & 124,78 & $4 \%$ \\
1923 & 19 & 2,66 & 125,47 & $10 \%$ \\
\hline
\end{tabular}

\section{CUADRO 12}

Empresas «varias» 1914-1923

\begin{tabular}{ccccc}
\hline Año & $N^{\circ}$ & Beneficios liquidos & Capital + reservas & Ben $/ c .+r$ \\
\hline 1914 & 1 & 0,00 & 0,37 & $0 \%$ \\
1915 & 2 & 0,01 & 0,53 & $2 \%$ \\
1916 & 2 & 0,00 & 0,62 & $0 \%$ \\
1917 & 5 & 0,03 & 1,48 & $1 \%$ \\
1918 & 7 & 0,27 & 1,79 & $14 \%$ \\
1919 & 7 & 0,17 & 1,84 & $15 \%$ \\
1920 & 10 & 1,02 & 3,87 & $32 \%$ \\
1921 & 10 & 0,05 & 4,72 & $4 \%$ \\
1922 & 10 & 0,20 & 4,76 & $6 \%$ \\
1923 & 11 & 0,14 & 4,19 & $5 \%$ \\
\hline
\end{tabular}




\section{BIBLIOGRAFÍA}

Alonso Olea, E. J. (1994): «Concierto Económico y Fiscalidad privilegiada: el uso del “paraíso fiscal” vizcaína. 1878-1937», en MARTín ACEÑA, P., y GARATE OJANGUREN, M. (Eds.), Economia y empresa en el norte de España (Una aproximación bistórica). San Sebastián, Diputación Foral de Gipuzkoa-U.P.V.

- 1995): El Concierto Económico (1878-1937). Origenes y formación de un Derecbo bistórico. Oñate: Instituto Vasco de Administración Pública.

DiPUTACIÓN DE ÁlAVA (1927): Reglamento del Impuesto sobre Utilidades de la riqueza mobiliaria de Alava. Vitoria: Impr. Provincial.

- (1928): Reglamento del Impuesto sobre las utilidades de la riqueza mobiliaria. Vitoria: Impr. Provincial.

DIPUTACIÓN DE GUIPÚZCOA (1929): Reglamento y tarifas para la aplicación y administración de la Contribución de Utilidades en Guipúzcoa. San Sebastián: Impr. Provincial.

DiPUTACIÓN DE VIZCAYA (1935): Instrucción para la imposición y cobranza del impuesto de Utilidades. Tarifa $1^{a}$, que grava las utilidades del trabajo personal. Bilbao: Impr. Provincial.

Diputación Foral y Provincial de Navarra (1928): Contribución de utilidades. Reglamento y Tarifas para su aplicación y administración. Año 1928. Pamplona: Impr. Provincial.

Estadisticas bistóricas de España. siglos XIX-XX (1985): Madrid: Fundación Banco Exterior.

EzCuRDIA, L. (1933): La contribución de utilidades en el Concierto Económico. San Sebastián: Nueva Editorial.

FRIG()LA PalaO, F. (1914): Hacienda local. El repartimiento de Utilidades. Manual de estudio. Bilbao.

GonzÁlez Portilla, M. (1994). «A.H.V. Expansión y crisis de la siderurgia española. La fractura de la Primera Guerra Mundial», en Martin ACENA, P., y Garate Ojanguren, M. (Eds.). Economia y empresa en el norte de España (Una aproximación bistórica). San Sebastián, Diputación Foral de Gipuzkoa-U.P.V.

Migoya, J. Y. E. (1910): La nueva tributación directa en Vizcaya. Bilbao: Casa de Misericordia.

MINISTERIO DE HACIENDA (1920): Ley reguladora de la Contribución sobre las utilidades de la riqueza mobiliaria, texto refundido de 19 de octubre de 1920. Madrid: M. Minuesa.

Ministerio de la GOBERnACIÓN (1913): Adición al Concierto de las Provincias Vascongadas en virtud del R.D. de 23 de $X$ de 1913. Aumento de 90.000 pts por el impuesto de Utilidades que satisfará la Diputación Provincial de Vizcaya. Datos que sirvieron para el vigente concierto del año 1906, el importe de las contribuciones concertadas y lo que recaudan las Diputaciones según los presupuestos de gastos é ingresos de las provincias de Vizcaya, Guipúzcoa y Alava. Madrid: Impr. Fabrica Nal. de la Moneda y Timbre.

MONTERO, M. (1995): La Californa del hierro. Las minas y la modernización económica y social de Vizcaya. Bilbao: Beitia.

RE(iLAMENTO (1901): Reglamento para la administración y cobranza de la contribución industrial y de comercio, aprobado por $R D$ de 28-5-1896 y modificado por la ley de 28-11-1899, estableciendo el año natural, por la de Utilidades de 27-5-1900 y por diferentes $R O$ dictadas basta el 21-9-1901. Madrid. 